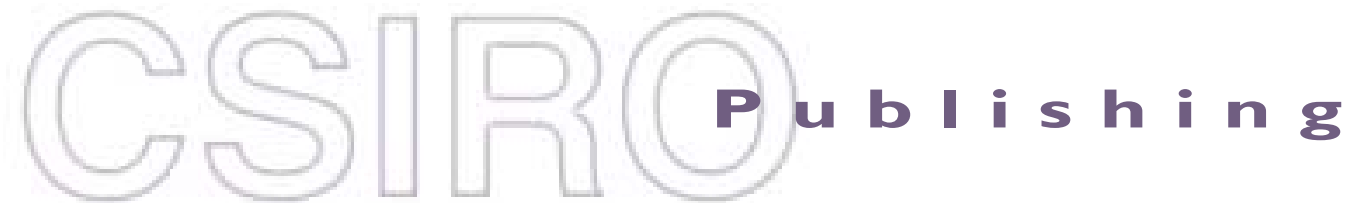

\section{Publications of the Astronomical Society of Australia}

Volume 19, 2002

(C) Astronomical Society of Australia 2002

An international journal of astronomy and astrophysics

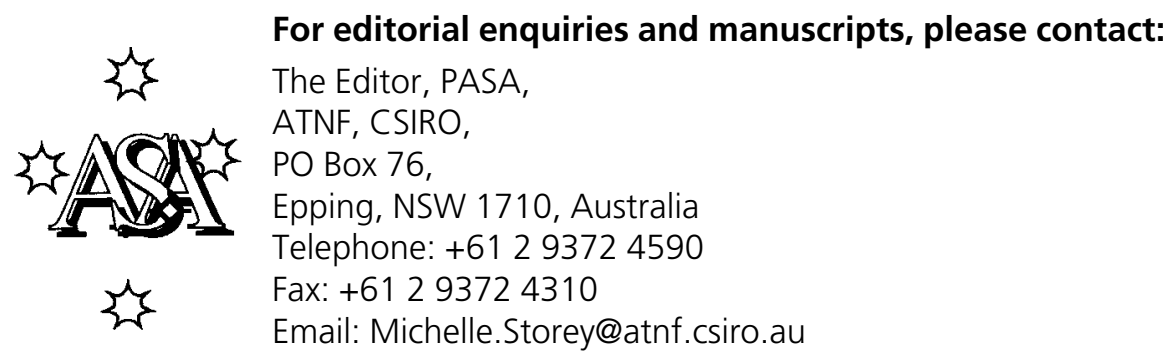

For general enquiries and subscriptions, please contact: CSIRO Publishing PO Box 1139 (150 Oxford St)

Collingwood, Vic. 3066, Australia

Telephone: +6139662 7666

Fax: +61 396627555

Email: publishing.pasa@csiro.au

C S I RO

PUBLISHING Published by CSIRO Publishing

for the Astronomical Society of Australia

www.publish.csiro.au/journals/pasa 


\title{
Brightness Temperature Constraints to Compact Synchrotron Source Radiation Obtained from IDV and VLBI Observations
}

\author{
K. I. Kellermann \\ National Radio Astronomy Observatory, 520 Edgemont Road, Charlottesville, VA 22903, USA \\ kkellerm@nrao.edu \\ Received 2001 September 25, accepted 2001 November 7
}

\begin{abstract}
We discuss the observational and theoretical constraints on the brightness temperature of compact opaque synchrotron sources. We consider the implication of observed apparent velocities on the amount of Doppler boosting and compare this with values deduced from directly measured brightness temperatures from ground and space based VLBI observations, as well as the implications of intraday variability. We also discuss the maximum rest frame brightness temperature expected under conditions of both inverse Compton cooling and equipartition conditions.
\end{abstract}

Keywords: galaxies: active — quasars: general — galaxies: jets — radiation mechanisms: non-thermal

\section{Historical Background}

The problems implied by the observations of intraday variability are not new. However, the new observations strain the interpretation so the arguments and models have become more sophisticated and correspondingly more complex.

The first reports of variability in extragalactic radio sources came from the Soviet Union in 1965. Gnadi Sholomitsky, a young Russian student, announced that he had detected $30 \%$ variations over a month in the $30 \mathrm{~cm}$ flux density of the quasar CTA 102 (Sholomitsky 1965, 1966). But these unexpected results were not generally accepted in the West. No information was given on the radio telescope or other instrumentation used for the observations, and the quality of the published presentation was poor. No one, including our group at Caltech, was able to confirm the presence of any variations in CTA 102. The credibility of the Soviet paper was not helped by the claim that the variations in CTA 102 were periodic with a 102day period, which prompted speculation by Shklovsky and Kardashev, as reported in Pravda, that CTA 102 might be an artificial transmission from an extraterrestrial civilisation.

The biggest problem, however, the one we are still struggling with, was that such rapid variations appeared to be theoretically impossible! Causality arguments placed an upper limit on the linear size of about 0.1 light years. CTA 102 together with CTA 21 were the first examples (Kellermann et al. 1962) of what was later called a Gigahertz Peaked Spectrum radio source with a spectral maximum near $1 \mathrm{GHz}$. Interpreting the low frequency spectral cutoff as synchrotron self absorption meant that the angular size had to be about 0.01 as. Combined with the linear size limited to 0.1 light years deduced from the observed flux density variations meant that CTA 102 could not be more distant than a few megaparsecs. But CTA 102 was known to have a measured redshift of 1.0.
Many years later we learned that Sholomitsky had used a military antenna system that was primarily used to track Soviet spacecraft, and understandably, he was not allowed to disclose the details of his observational program. We now know that CTA 102 does vary at decimetre wavelengths (e.g. Hunstead 1972) at about the amount reported by Sholomitsky, although there is no evidence of cyclical changes. The story about extraterrestrial signals was just a spoof by Shklovsky and Kardashev, which was taken too seriously by the Russian reporters who, like all reporters, were hungry for a story.

The reality of rapid flux density variations was soon confirmed by Dent (1965) and by Pauliny-Toth \& Kellermann (1966). 3C 120 and 3C 273 were found to vary by as much as $10 \%$ in a few days, or more than $1 \%$ within a day. In 1966, the flux density of 3C 273 appeared to increase by $50 \%$ in six months corresponding to a change in luminosity of $10^{45} \mathrm{erg} \mathrm{sec}^{-1} \mathrm{yr}^{-1}$ equivalent to turning on Cygnus $\mathrm{A}$ in less than a year. Significant flux density changes were found at wavelengths as long as $40 \mathrm{~cm}$ (Pauliny-Toth \& Kellermann 1966) and $70 \mathrm{~cm}$ (Fanti et al. 1979) — a phenomenon that later became known as low frequency variability. Later Heeschen (1984) and Heeschen et al. (1987) showed that day-to-day variations of a per cent or so were common, a phenomenon he called flickering, and what we now call intraday variability (IDV). However, Heeschen \& Rickett (1987) noted that the observed rapid variability might not be intrinsic, but instead due to interstellar scintillation. But, whether IDVs are due to scintillation or are intrinsic, the interpretation of rapid variability, especially at longer wavelengths, remains a challenge even today. Although the effects of relativistic beaming, as evidenced by observations of superluminal motion can, in principle, account for the observed time scales and the corresponding apparent high brightness temperatures, problems still remain. 


\section{Relativistic Beaming and Superluminal Motion}

The twin relativistic jet model of Blandford \& Rees (1974) postulates an intrinsic two-sided relativistic jet which has three observational consequences:

i) The Doppler frequency shift of the observed radiation is given by

$$
\delta=\gamma^{-1}(1-\beta \cos \theta)^{-1}
$$

where $\delta$ is referred to as the Doppler factor, $\gamma$ is the Lorentz factor defined by $\gamma=\left(1-\beta^{2}\right)^{-1 / 2}, \beta=v / c$ is the velocity of the relativistic flow in units of the speed of light, and $\theta$ is the angle between the jet and the line of sight.

ii) Due to relativistic aberration, the apparent transverse velocity of motion is given by

$$
\beta_{\text {app }}=\beta_{\mathrm{p}} \sin \theta /\left(1-\beta_{\mathrm{p}} \cos \theta\right)
$$

where $\beta_{\mathrm{p}}$ is the pattern velocity describing the actual component motion. The apparent transverse velocity of a component approaching close to line of sight reaches a maximum value of $\gamma c$ at an angle $\theta \sim 1 / \gamma$ to the line of sight. For the receding component, the limiting velocity is $c / 2$.

iii) Due to relativistic beaming, the apparent flux density, $S$, of a moving component is enhanced over its stationary value $S_{0}$, by an amount

$$
S / S_{0}=\delta^{a+\alpha},
$$

where $a$ is 2 or 3 depending on the geometry, $\alpha$ is the spectral index defined by $S=v^{\alpha}$, and the value of $\delta$ is determined by the bulk motion, $\beta_{\mathrm{b}}$, of the relativistic flow.

\section{The Problems}

The observed changes of flux density with time were easily interpreted in terms of a simple adiabatically expanding source model (Shklovsky 1965; Pauliny-Toth \& Kellermann 1966; van der Laan 1966). However, there were serious problems with this simple picture which has now been replaced by more sophisticated shocks in relativistic jets (e.g. Marsher \& Gear 1985; Hughes, Aller, \& Aller 1989).

Hoyle, Burbidge, \& Sargent (1966) were quick to argue from causality considerations that the observed rapid variability implied such small linear dimensions, that if the quasar redshifts were interpreted as a measure of distance, the relativistic electron population would be rapidly extinguished by inverse Compton scattering. Kellermann $\&$ Pauliny-Toth (1969) put these arguments on a quantitative observational basis showing that due to inverse Compton cooling, the maximum sustainable brightness temperature for an incoherent source of synchrotron radiation is about $10^{12} \mathrm{~K}$. The detection of significant variations at decimetre wavelengths and the observations of intraday variability later exacerbated the problem. However,
Woltjer (1966) and Rees $(1966,1967)$ pointed out that if there is a relativistic bulk motion, the radiation is beamed along the direction of motion, and the cross-section for inverse Compton scattering is greatly reduced.

Even before the discovery of radio variability, Shklovsky (1964) speculated that differential Doppler boosting might explain the one sided appearance of the M87 jet. The introduction of the twin jet model of Blandford \& Rees (1974) and the subsequent discovery of superluminal motion in quasars and AGN appeared at first to confirm the importance of relativistic beaming (Cohen et al. 1971; Whitney et al. 1971). But it was difficult at first to understand the large number of apparent superluminal sources since only a small fraction oriented close to the line of sight is expected to exhibit superluminal velocities (Cohen et al. 1977).

This presented a paradox. The a priori probability of observing a source within an angle $\theta$ to the line of sight is of the order $1 / \theta^{2}$, corresponding to a probability of $1 / \gamma^{2}$ for observing an apparent velocity $\gamma c$. In a randomly oriented sample, the motion will mostly be oriented close to the plane of the sky, and the apparent projected velocity of most sources should be close to the speed of light as foreshortening effects will be small. But about half of all observed compact radio sources showed superluminal motion with apparent velocities more than $5 c$.

It was quickly realised that as a result of 'Doppler' or 'relativistic' boosting, flux limited samples should preferentially contain components moving toward the observer. Receding or stationary components appear much weaker, so most sources appear as one sided. However, it was still a puzzle to understand why the approaching, and stationary or receding, components appeared to have comparable flux densities, since equation (3) shows that differential Doppler boosting should cause a flux density ratio of at least $100-1000$ for $5 \leq \gamma \leq 10$.

Blandford \& Konigl (1979) suggested that one of the components might be the stationary nozzle of the approaching jet at the point where it changes from optically thick to optically thin, so that the flux density of this 'core' component as well as the moving component is also Doppler boosted. Consideration of differential Doppler boosting led to the concept of unified models, which is frequently invoked to understand the observed differences in the properties of quasars, AGN, and BL Lac objects in terms of the orientation of the relativistic beam and an obscuring torus with respect to the line of sight.

For simple ballistic motion where $\beta_{\mathrm{p}}=\beta_{\mathrm{b}}$ equations (2) and (3) predict a simple relation between the observed transverse velocity and the Doppler boosting, and thus provide a quantitative test of the model. If both the approaching and receding components are observed, then both $\beta$ and $\theta$ are uniquely determined. However, the analysis of the distribution of observed motions in a sample of compact radio sources selected from the literature indicated that either $\beta_{\mathrm{p}} \neq \beta_{\mathrm{b}}$ or that there is a broad distribution in the intrinsic value of $\gamma$ among sources (Vermeulen \& Cohen 1994; Lister \& Marsher 1997). A later analysis by 
Vermeulen (1995) of a larger sample of sources suggested that the early samples were biased in the sense that primarily sources with previously detected rapid motions were observed at multiple epochs and reported in the literature.

\section{The New Observations}

Since 1995, Rene Vermeulen, Marshall Cohen, Anton Zensus and I have been observing a sample of radio galaxies and quasars with the VLBA in order to better define their motions. We want to know:

- Where does the relativistic flow get accelerated and collimated to form jets?

- Does the flow follow curved or straight trajectories?

- Do different components within a jet follow the same or different trajectories? Do they have the same or different speeds?

- Are there accelerations or decelerations?

- Is the apparent velocity related to luminosity, X-ray or gamma-ray emission? Or to anything else?

- Is the time of appearance of a new component emerging from the nucleus related to the start of a flux density outburst?

- Are there any observed differences in the kinematics of radio galaxies, quasars, or BL Lac objects?

- Are the simple ballistic models correct, or are there differences between the bulk flow velocity and the pattern velocity as might be expected if the observed motions are due to the propagation of shocks rather than the flow of material (e.g. Vermeulen \& Cohen 1994)?

- What is the distribution of intrinsic Lorentz factors?

- Are the observed Lorentz factors sufficiently large to explain the high brightness temperatures deduced from the observations of intraday variability?

Some preliminary results of our observations have already been published (Kellermann et al. 1998, 1999, 2000). It is perhaps curious that the observed flow is almost always outward, away from the apparent central engine, yet it is widely thought that the source of energy is infall onto a massive black hole. But there is also evidence in some sources for an inflow, characteristic of reverse shocks. Often the trajectory is curved, especially close to the nucleus. Generally, each component moves with constant speed until it fades away, and each component appears to move along a similar trajectory. However, in some carefully studied sources, such as $3 \mathrm{C} 345$, there is evidence for small accelerations and slightly different trajectories (e.g. Lobanov \& Zensus 1999).

As discussed above, provided that $\beta_{\mathrm{p}}=\beta_{\mathrm{b}}$, observations of the apparent component velocity and the ratio of flux densities of approaching and receding components can, in principle, be used to solve uniquely for $\beta$ and $\theta$. In this case, in a flux density limited sample, the combined effect of solid angle selection and Doppler boosting leads to a strong bias toward sources with their beams close to the critical angle $1 / \gamma$. In this case most sources will have an apparent velocity very close to $\gamma c$ (Vermeulen $\&$
Cohen 1994). On the other hand, if Doppler boosting is not important, then in any flux limited sample most sources will lie close to the plane of the sky and show apparent velocities close to the speed of light, with only a small tail extending toward $\gamma c$. Surprisingly, in our sample of about 100 strong compact AGN and quasars, we find most sources have an apparent velocity only a few times the speed of light with a small tail extending up to about $10 c$ (Kellermann et al. 2000), consistent with little or no Doppler selection.

How do we reconcile the difference between the observed and predicted distributions? Possibly, there is a spread in intrinsic velocity (e.g. Lister \& Marsher 1997). Alternately, the bulk flow velocity may be much less than the pattern velocity, so that there is relatively little Doppler bias in favour of observing beams, which are oriented close to the critical angle. Indeed, the observed distribution of apparent velocity is not unlike the simple light echo model discussed by Ekers \& Laing (1990) where there is no Doppler bias, and the observed distribution depends only on the orientation of the relativistic beam.

\section{The Brightness Temperature Problem}

The effect of inverse Compton scattering is usually expressed as an upper limit on the brightness temperature. Under equilibrium conditions, the maximum brightness temperature of a stationary incoherent source of synchrotron radiation from relativistic electrons is about $10^{12} \mathrm{~K}$ (Kellermann \& Pauliny-Toth 1969). Readhead (1994), Begelman, Rees, \& Sikora (1994), and Guijosa \& Daly (1996) have argued that if there is equipartition between the magnetic energy density and relativistic particle density, then the peak brightness temperatures are nearly an order of magnitude lower. The concept of equilibrium between particle and magnetic energies was introduced long ago to minimise the apparent excessive energy content in extended radio galaxies with characteristic ages of $10^{8}-10^{9} \mathrm{yr}$ (Burbidge 1959). However, there is no reason to expect equipartition conditions to exist in sources where there is clearly a sudden highly collimated violent release of relativistic plasma. More likely, in these sources with typical ages of only 1-100 yr estimated from variability observations, there is likely to be an excess of particle over magnetic energy.

In principle, the energy contained in relativistic particles and magnetic fields can be determined directly from observations of flux density, self absorption cutoff frequency, and angular size. Although the uncertainties are very large as the calculated energies depend on a large power of the measured quantities, observational results often indicate a large ratio of particle to magnetic energy, consistent with brightness temperatures limited by inverse Compton cooling (e.g. Hornby \& Williams 1966; Williams 1966; Bridle 1967; Kellermann \& Pauliny-Toth 1969).

What are the observational constraints to the brightness temperature? Ground based observations with the VLBA show typical apparent maximum brightness temperatures 
of $10^{11}-10^{12} \mathrm{~K}$, just in the range expected from the inverse Compton limit (Kellermann et al. 2000). For a few sources, our $2 \mathrm{~cm}$ observations indicate a lower limit to the observed brightness temperature of as much as $10^{13} \mathrm{~K}$ stretching the inverse Compton limit for a stationary synchrotron source. But the maximum measurable brightness temperature depends only on the flux density and interferometer baseline length, independent of wavelength. For a typical flux density of a few janskys and baseline lengths of $10000 \mathrm{~km}$, this corresponds to about $10^{12} \mathrm{~K}$ so that it is not possible to obtain a stringent test of the inverse Compton limit using interferometer baselines on the surface of the Earth.

VLBI observations using orbiting radio telescopes in conjunction with ground based radio telescopes can increase the brightness temperature limit beyond that which is obtained using terrestrial baselines alone. Observations made with a NASA TDRSS satellite (Linfield et al. 1989) and the Japanese space VLBI satellite, HALCA (Hirabayashi et al. 2000), in conjunction with the VLBA and other ground based arrays have suggested rest frame brightness temperatures well in excess of the $10^{12} \mathrm{~K}$ inverse Compton limit (Tingay et al. 2001).

However, the observations of intraday variability appear to require much higher brightness temperatures. Using the same causality arguments employed by Sholomitsky and others in the 1960s, Kedziora-Chudczer et al. (1997, 2001) and Dennett-Thorpe \& de Bruyn (2000) claim brightness temperatures as high as $10^{21} \mathrm{~K}$ for the most rapid variables which show changes on time scales $<1 \mathrm{~h}$.

These light travel time arguments are, of course, valid only if the observed variations are intrinsic and not due to propagation effects. Although the apparent correlation of radio and optical variations argue in favour of this interpretation (Wagner \& Witzel 1995), recent observations of seasonal modulation and time delays provide convincing evidence that the most rapid variability is due to interstellar scintillation and is not intrinsic (Jauncey et al. 2000). However this does not eliminate the puzzle of understanding the apparent excess brightness temperatures as only sources smaller than about $10 \mu$ as will scintillate due to irregularities in the interstellar medium. This corresponds to brightness temperatures in excess of $10^{15} \mathrm{~K}$, still well above the inverse Compton limit.

\section{How do we explain these apparent excessive brightness temperatures?}

Relativistic Beaming: The most common interpretation of the high brightness temperatures inferred by direct measurement or implied by IDV invokes the effect of relativistic beaming. From the directly measured lower limits of $10^{13} \mathrm{~K}$ from ground based observations with the VLBA and from space VLBI, Doppler factors of the order of 10 are needed to explain the observed brightness temperatures. This is consistent with our VLBA observations of radio source kinematics. However, IDVs imply much larger Doppler factors, far in excess of what is directly observed.
If IDV is intrinsic, then including both the flux density enhancement and the effect of time dilation, relativistic beaming increases the observed brightness temperature by a factor of $\delta^{3}$ to $10^{21} \mathrm{~K}$, so that Doppler factors of up to 1000 are required to reduce the rest frame brightness temperature below the Compton limit of $10^{12} \mathrm{~K}$. If IDV is due to propagation effects, relativistic beaming increases the apparent brightness temperature by a factor of $\delta$, so that a Doppler factor of 1000 is still needed to reduce the apparent temperature of $10^{15} \mathrm{~K}$ below the inverse Compton limit.

Thus whether IDVs are intrinsic or due to propagation effects, Doppler factors up to 1000 are needed to reduce the observed brightness temperature to the inverse Compton limit. However, there is no evidence from observations of component motions that such large Doppler factors exist. Nor does it seem theoretically possible to sustain such high Doppler factors (Begelman et al. 1994).

Coherent Mechanisms: The limit of $10^{12} \mathrm{~K}$ applies only to incoherent synchrotron radiation. Various authors (e.g. Colgate 1967; Melrose 1999) have discussed different coherent or collective processes, including coherent plasma waves and stimulated synchrotron emission (synchrotron masers). A necessary condition for maser activity or negative absorption is an inverted electron energy distribution (Twiss 1958) but there has been no consensus as to whether or not synchrotron maser activity can occur in practice (e.g. Bekefi \& Brown 1961; Wild et al. 1963; McCray 1966; Zheleznyakov 1966, 1967; Benford 1992; Lesch \& Pohl 1992; Benford \& Tzach 2000; Zheleznyakov et al. 2000). Although various absorption mechanisms such as induced Compton scattering or Raman scattering (e.g. Sunyaev 1971; Wilson 1982; Begelman et al. 1994; Coppi, Blandford, \& Rees 1993; Sincell \& Krolik 1994; Levinson \& Blandford 1995) appear to limit the observed brightness temperature of a coherent source, Benford \& Lesch (1998) argue that conventional theory does not adequately explain laboratory experiments or, indeed, even observed pulsar radiation.

Non Stationary Solutions: As pointed out in our original paper (Kellermann \& Pauliny-Toth 1969), the widely discussed inverse Compton limit of $10^{12} \mathrm{~K}$ is valid only after the source has reached equilibrium following the injection or acceleration of relativistic electrons into a magnetic field. For a limited period after relativistic electrons start to radiate, the brightness temperature can exceed the inverse Compton limit for a period of the order of the Compton decay time. The lifetime due to first order inverse Compton is easy to calculate as it is mathematically equivalent to synchrotron radiation losses. Following Slysh (1992), at $5 \mathrm{GHz} T_{B}$ can remain $>10^{15} \mathrm{~K}$ for about an hour, more or less what is observed. However, it is more difficult to calculate the lifetime including the effects of higher order scattering taking into account the smaller Klein-Nishina cross-section for high order scattering as well as possible replenishment of the electron population due to pair production. More work is needed on this problem. Also, of course, if the supply of relativistic particles continues 
or the relativistic electrons are continually reheated, then an arbitrarily high brightness temperature can be maintained indefinitely, provided that the relativistic particle energy distribution extends to sufficiently large energies (Kellermann \& Pauliny-Toth 1969; Pacini \& Salvati 1974).

Proton Synchrotron Emission: Synchrotron radiation from relativistic protons can have a brightness temperature which exceeds that from electron radiation by a factor of $\left(m_{p} / m_{e}\right)^{9 / 7}$, which is roughly consistent with that deduced from IDV without the need to invoke relativistic beaming (Jukes 1967; Pacini \& Rees 1970; Kardashev 2000; Aharonian 2001). However, the required magnetic field strength is much larger by a factor of $\left(m_{p} / m_{e}\right)^{2}$.

\section{Commentary}

While this conference has not provided answers to any questions, perhaps the questions have been refined and are now somewhat more focused.

From the observations of time delays and seasonal modulation reported by Jauncey et al. (2000) and by Kedziora-Chudczer (2002) it appears that there is now overwhelming evidence that propagation effects play an important role in intraday variability. But we still do not know if IDVs are entirely due to propagation effects or if there are also intrinsic effects as suggested by polarisation observations reported by Gabuzda (2002) and Gabuzda et al. (2000). If due to propagation, where is the screen? Are the reported radio-optical correlations real? What can we learn from polarisation observations?

Is the maximum brightness temperature limited by inverse Compton radiation to $10^{12} \mathrm{~K}$ as suggested by Kellermann \& Pauliny-Toth (1969), or is it closer to the $10^{11} \mathrm{~K}$ equilibrium value claimed by Readhead (1994) if there is equilibrium between the particle and magnetic energies. While equilibrium conditions may apply for lobes and kiloparsec scale jets which may be $10^{8}-10^{9} \mathrm{yr}$ old, it is not clear if these arguments can be applied to compact sources which may only be $1-100 \mathrm{yr}$ old and expanding or moving relativistically. However, if the brightness temperature is limited by equipartition arguments, then the required Lorentz factors to explain the high apparent brightness temperatures inferred from the IDV observations are correspondingly increased. If the former, is self Compton or external Compton scattering more important? Is the inferred excess of particle energy over magnetic energy really a problem, or is it consistent with the observation of shock or bulk motion with velocities near the speed of light?

Are the classical incoherent synchrotron models satisfactory? If so what Doppler (Lorentz) factors are required? Can we explain the high inferred brightness temperatures as transient radiation before Compton effects cool the radiation, or is there a continued supply of relativistic particles which maintain the high brightness temperature for longer than the Compton cooling time? Are coherent processes or proton synchrotron radiation important? Or is new physics required?

The new observations of radio IDV from the ATNF (Kedziora-Chudczer et al. 2001) and from Bonn have focused our attention on the problems and strain the theoretical models. The observation of high energy high order Compton scattered emission at X-ray, GeV, and TeV energies may be critical to understanding the importance of Compton cooling and relativistic beaming but are so far very limited in sensitivity, sky coverage, time resolution, and angular resolution.

We know very little about the luminosity function of $\gamma$-ray sources. Only about 50 are known and the range of observed flux densities between the strongest and weakest is only about a factor of 100 (Mattox, Hartman, \& Reimer 2001). We do not know if the number of $\gamma$-ray detections will continue to increase or if the distribution is bimodal as it is for the radio emission and there is a class of $\gamma$-ray quiet quasars. There are only two TeV detections (Mrk 421 and Mrk 501) so we know very little about their spatial distribution or luminosity function, but both of these sources are relatively nearby. The all sky coverage of the Japanese MAXI X-ray observatory will allow the observation of X-ray flares without the need to know in advance where to point. The new data that will become available such as from HESS and VERITAS at TeV energies, from GLAST at $\gamma$-ray energies, and from Constellation-X at $\mathrm{X}$-ray energies will tell us something, but what?

Space VLBI observations with baselines of $100000 \mathrm{~km}$ or more can directly confirm the existence of brightness temperatures up to the $10^{14}-10^{15} \mathrm{~K}$ inferred from IDV observations. But directly imaging IDVs will be difficult as conventional Fourier synthesis techniques require observations over many hours during which time the source structure of IDVs will obviously change. New techniques will be required to image IDVs.

\section{Acknowledgments}

I thank my colleagues Rene Vermeulen, Anton Zensus, and Marshall Cohen who have collaborated on some of the research reported here. Section 6 is based on the summary remarks made at the conference by this author and the ensuing heated debate. We did not go home from the workshop with answers, but we have focused our attention on the questions and how we might get the answers. None of the issues are new. The arguments are not new, but the new observations from across the entire electromagnetic spectrum have sharpened the arguments which are now more sophisticated, although a pessimist might say, 'As they are now described by more parameters they have lost their predictability and thus their attractiveness'.

We all thank the participants who provided the fuel for these discussions and the organisers, particularly Lucyna Kedziora-Chudczer and Dave Jauncey who gave us the opportunity to exchange data on new observations, planned new facilities, and the theoretical basis to try to understand the implications of what we have observed. 
The extraordinary winter weather we experienced in Sydney was a pleasant contrast to the participants coming from the hot humid American and Japanese summer and the cold damp European summer.

I thank the two anonymous referees for their careful reading of the manuscript and their suggestions which have greatly improved this paper.

\section{References}

Aharonian, F. A. 2001, preprint (astro-ph/0106037)

Begelman, M. C., Rees, M. J., \& Sikora, M. 1994, ApJ, 429, L5

Bekefi, G., \& Brown, S. C. 1961, AmJPh, 29, 404

Benford, G. 1992, ApJ, 391, L59

Benford, G., \& Lesch, H. 1998, MNRAS, 301, 414

Benford, G., \& Tzach, D. 2000, MNRAS, 317, 497

Blandford, R., \& Konigl, A. 1979, ApJ, 232, 34

Blandford, R., \& Rees, M. 1974, MNRAS, 169, 395

Bridle, A. 1967, Obs, 87, 263

Burbidge, G. 1959, ApJ, 129, 849

Cohen, M., et al. 1971, ApJ, 170, 207

Cohen, M. H., et al. 1977, Nature, 268, 405

Colgate, S. A. 1967, ApJ, 150, 163

Coppi, P., Blandford, R. D., \& Rees, M. J. 1993, MNRAS, 262, 603

Dennett-Thorpe, J., \& de Bruyn, A. G. 2000, ApJ, 529, L65

Dent, W. 1965, Science, 148, 1458

Ekers, R., \& Laing, H. 1990, in Parsec Scale Radio Jets, eds J. Zensus, \& T. Pearson (San Francisco: ASP), 333

Fanti, R., Ficarra, A., Mantovani, F., Padrielle, R., \& Weiler, K. 1979, A\&AS, 36, 359

Gabuzda, D. C. 2002, PASA, 19, in press

Gabuzda, D. C., Kochenkov, P. Yu., Cawthorne, T. V., \& Kollgaard, R. I. 2000, MNRAS, 313, 627

Guijosa, A., \& Daly, R. A. 1996, ApJ, 461, 600

Heeschen, D. S. 1984, AJ, 89, 111

Heeschen, D. S., \& Rickett, B. J. 1987, AJ, 89, 1111

Heeschen, D. S., Krichbaum, T., Schalinski, C. J., \& Witzel, A. 1987, AJ, 94, 1493

Hirabayashi, H., et al. 2000, PASJ, 52, 955

Hornby, J. M., \& Williams, P. J. S. 1966, MNRAS, 131, 237

Hughes, P. A., Aller, H. D., \& Aller, M. F. 1989, ApJ, 341, 54

Hunstead, R. W. 1972, MNRAS, 157, 367

Hoyle, F., Burbidge, G., \& Sargent, W. 1966, Nature, 209, 751

Jauncey, D., et al. 2000, in Astrophysical Phenomena Revealed by Space VLBI, eds H. Hirabayashi, et al. (Sagamihara, Japan: Inst. of Space and Astronaut. Science), 147

Jukes, J. D. 1967, Nature, 216, 461

Kardashev, N. S. 2000, AZh, 77, 813 (ARep, 44, 1063)

Kedziora-Chudczer, L. 2002, private communication
Kedziora-Chudczer, L., et al. 1997, ApJ, 490, L9

Kedziora-Chudczer, L., et al. 2001, MNRAS, 325, 1411

Kellermann, K., \& Pauliny-Toth, I. 1969, ApJ, 155, L71

Kellermann, K. I., Long, R. J., Allen, L. R., \& Moran, M. 1962, Nature, 195, 692

Kellermann, K. I., Vermeulen, R., Zensus, A., \& Cohen, M. 1998, AJ, 115, 1295

Kellermann, K. I., Vermeulen, R., Zensus, A., Cohen, M. H., \& West, A. 1999, NewAR, 43, 757

Kellermann, K. I., Vermeulen, R., Zensus, A., \& Cohen, M. H. 2000, in Astrophysical Phenomena Revealed by Space VLBI, eds H. Hirabayashi, et al. (Sagamihara, Japan: Inst. of Space and Astronaut. Science), 159

Lesch, H., \& Pohl, M. 1992, A\&A, 254, 29

Levinson, A., \& Blandford, R. 1995, MNRAS, 274, 717

Linfield, R., et al. 1989, ApJ, 336, 1105

Lister, M., \& Marsher, A. 1997, ApJ, 476, 572

Lobanov, A., \& Zensus, A. 1999, ApJ, 521, 509

Marsher, A., \& Gear, W. 1985, ApJ, 298, 114

Mattox, J. R., Hartman, R. C., \& Reimer, O. 2001, ApJS, 135, 155

McCray, R. 1966, Science, 154, 1323

Melrose, D. B. 1999, Ap\&SS, 264, 391

Pauliny-Toth, I. I. K., \& Kellermann, K. I. 1966, ApJ, 155, L71

Pacini, F., \& Rees, M. 1970, Nature, 226, 622

Pacini, F., \& Salvati, M. 1974, ApJ, 188, L55

Readhead, A. 1994, ApJ, 426, 51

Rees, M. 1966, Nature, 211, 468

Rees, M. 1967, MNRAS, 135, 345

Shklovsky, I. S. 1964, AZh, 40, 972 (SvA, 7, 748)

Shklovsky, I. S. 1965, AZh, 42, 30 (SvA, 7, 748)

Sholomitsky, G. 1965, AZh, 42, 673 (SvA, 9, 516)

Sholomitsky, G. 1966, Astron. Tzirk., No. 359

Sincell, M. W., \& Krolik, J. H. 1994, ApJ, 430, 550

Slysh, V. 1992, ApJ, 391, 453

Sunyaev, R. A. 1971, AZh, 48, 224 (SvA, 15, 190)

Tingay, S. J., et al. 2001, ApJ, 549, L58

Twiss, R. Q. 1958, AuJPh, 11, 565

van der Laan, H. 1966, Nature, 211, 1131

Vermeulen, R. 1995, Proc. Nat. Acad. Sci., 92, 11385

Vermeulen, R., \& Cohen, M. 1994, ApJ, 430, 467

Wagner, S., \& Witzel, A. 1995, ARA\&A, 33, 163

Whitney, A., et al. 1971, Science, 173, 225

Wilson, D. B. 1982, MNRAS, 200, 881

Wild, P., et al. 1963, ARA\&A, 1, 291

Williams, P. J. S. 1966, Nature, 210, 285

Woltjer, L. 1966, ApJ, 146, 597

Zheleznyakov, V. 1966, Soviet Physics JETP, 51, 570

Zheleznyakov, V. 1967, SvA, 11, 33

Zheleznyakov, V., et al. 2000, in Radio Astronomy at Long Wavelengths, eds R. Stone, et al. (Washington: American Geophysical Union), 57 